\title{
The Analysis of the Adjustment Mechanism of Health-care Qigong to Human Emotion Based on the Theory of Mental Energy
}

\author{
Yunyan Zhong \\ Sichuan University of Science and Engineering, Zigong, 643000, China
}

Keywords: The theory of mental energy. Health Qigong. Adjustment of human emotion

\begin{abstract}
During the development and evolution of Chinese Qigong for thousands of years, it was combined with the essence of Chinese traditional philosophy, literature, the theory of Chinese medicine and religion, etc. In recent years, health-care Qigong appears on the basis of Qigong. A brief introduction of health-care Qigong is given in this thesis in which the effect of emotional adjustment made by health-care Qigong is shown from different aspects such as the adjustment of human emotion, improvement of the function of central nervous system and cultivation of morality. Meanwhile, in terms of imagine characteristics, culturology and psychology, the emotional adjustment mechanism of health-care Qigong is analyzed in this thesis og which the purpose is to provide reference for the study on the adjustment mechanism of health-care Qigong to human emotion based on the theory of mental energy.
\end{abstract}

\section{Introduction}

Health-care Qigong is a kind of national traditional sports which based on the combination of physical activities, inspiration and expiration and psychological adjustment. And health-care Qigong is also called a kind of self -motion. It is initiative exercise with its purpose of fitness. Health-care Qigong can effectively adjust human emotion, improve the function of central nervous system and cultivate morality. Therefore, human emotion can be adjusted effectively.

\section{The brief introduction to health-care Qigong}

Health-care Qigong is the skill of physical and mental exercise, which integrates the regulation of body, breathing and mind. The regulation of body, breathing and mind is called "the three regulation" in the science of Qigong. The science of Qigong defines the qualitative Qigong as "three in one”. All the physical and mental activities, which can realize "three in one", can be regarded as Qigong. And the basic characteristics of doing Qigong is to reach the state or condition of "three in one”. Qigong is a way of physical and mental practice as well. What's more, Qigong is featured with strong Chinese traditional characteristics, because Chinese traditional culture including Confucianism, Buddihism, Tanism, Medical and Kungfu plays an important role during the process of creation and development of Qigong. In recent years, health-care Qigong appears on the basis of Qigong. Health-care Qigong is a kind of national traditional sports which based on the combination of physical activities, inspiration and expiration and psychological adjustment. And health-care Qigong is also called a kind of self -motion. It is initiative exercise with its purpose of fitness. Health-care Qigong, pays more attention to operability and extensive use, meets the need of mass fitness and fits the core of the Outline of National Fitness Program. Due to the deep influence by Chinese traditional culture, the way to practice health-care Qigong is unique and the psychological factor counts in practice of health-care Qigong. In 2002, State General Administration of Health-care Qigong Management Center recreated four types of health-care Qigong. They are Yi jin jign, Wu qin xi, Liu zi jue and Ba duan jin. Yi jin jing mainly aims at strengthening tendons and bones so as to adjust the balance of the five internal organs(heart, liver, spleen, lungs and kidneys), develop the human potential and achieve the purpose of strong body and longer life through the rotation and flexion of spine, the movement of arms, legs and internal organs. Wu qin xi can keep the mind single-minded and effectively eliminated the distractions which are harmful to the physical and mental health. It can also create good inner 
environment to let trainers be peaceful by limitating the actions, expression and mood of tigers, deers, bears, apes and cranes. Liu zi jue applies inspiration and expiration coordinating with the pronunciation of Chinese letters $\mathrm{xu}$, he, hu, yan, chui and xi to adjust the heart, liver, spleen, lungs, kidneys and triple energizer in order to realize strong body, less illness and longer life. Just as its name implies, Ba duan jin divided Qigong into eight segments, and each of them has one different movement. It pursue the symmetry and all-body exercise, which can help trainers adapt to pressure, improve the function of nervous and humoral regulation and enhance the cardio and pulmonary function.

\section{The effect of health-care Qigong's emotional adjustment}

\section{The adjustment of human emotion}

Emotion is people's experience of the objective attitude to things. It is also the reaction to objective affairs and directly influences people's daily life, work and health. Pleasant and stable mood can not only effectively improve learning and work efficiency, but also can regulate the physical and mental functions. Therefore, people who have good mood can enjoy good health. However, emotion can cause negative effect such as physical or mental disease. Health-care Qigong is able to adjust human emotion, which is already vertified. A study on the emotional adjustment of college students who practice Ba duan jin by some scholars shows that the students can effectively ease tension, stress and depression though practicing Ba duan jim. The condition of their emotion can be well adjusted, while negative emotion such as hostility, obcession and paranoia can be improved. The main reason why Ba duan jin can effectively adjust human emotion is that trainers need to be peaceful and try to do meditation and follow conscious to make actions when they practice Ba duan jin. Health-care Qigong Ba duan jin can effectively consolidate nerve's flexibility and coordination, and at the same time alleviate brain fatigue, adjust emotion and keep physical and mental balance. Some scholars who study on Yi jin jing trainers found that Yi jin jing can well ease trainers' emotion of depression and anxiety. Whether a person has once practiced Yi jin jing badly impacts the trainers; happiness and anxiety self-assesment.

\section{Improve the function of central nervous system}

When people are practicing health-care Qigong, wave alpha increases to strengthen, then the initiative inhibition of cerebral cortex improves and disperses so as to change human sense and change vegetative nervous system which manage the movement of inner organs, on the basis of which the excitability of sympathetic nervous system is reduced and the excitability of parasympathetic nervous system is enhanced, so the functions of inner organs dominated by that nervous systems will be changed. Here comes the cerebral cortex entering into a state of low psychological load and low energy consumption. At the same time, when people are practicing health-care Qigong, the brain waves which are with longer cycle, higher amplitude, better synchronism and lower frequency are different with that when people are awake, sleeping or resting. With the simultaneous existence of wave theta and wave alpha, cerebral cortex cells can be better ordered if they are disordered caused by excessive excitability. The central nervous system can be relaxed because the ability of coordinating excitability and depression is increased. That's how the function of central nervous system is improved. And human emotion is adjusted effectively.

\section{Cultivating morality}

China is a county in which ethics and morality are paied great attention to. There's a record in " $\mathrm{Li}$ ji•Da xue": "things with high quality will be known to others; people will be with sincere heart to own one; the honest heart will make good ethics; a family is built on the basis of good ethics; then followed a great county; countries make a peaceful world". It is a must for a man with high morality to right his mind and perfect himself. As health-care Qigong inherits Chinese great tradition of emphasized morality, it requires trainers to be peaceful and optimistic and lay emphasis on cultivating morality and soft sentmental. Generally, health-care Qigong tend to advocate the idea that ethics is before skills and learn how to be polite before learn how to practice Kung fu. Trainers must have firmed faith and perseverance. What is more important is that trainers must be gender and avoid 
pursuit of fame. Health-care Qigong appeals to fit to nature and be indifferent to fame and wealth so that the trainers can keep good health and enjoy high morality.

\section{The emotional adjustment mechanism of health-care Qigong}

\section{The imagine characteristics of health-care Qigong}

The imagine characteristics of health-care Qigong are as follows: (1) the contents of imagination. The unity of form and mind is given priority to in the process of health-care Qigong. In the science of Qigong, form means the posetures and the forms of movement when people are doing Qigong. And mind refers to the under conscious, metal activities and thought and psychology. There is a big difference between health-care Qigong and ordinary mental work. Ordinary mental work is about the affairs which do not have anything with body, while, what the Qigong imagines is the while activities including body and mind, the flow of air and the process of application of power. (2) the principle of imagination. The core principle of practicing health-care Qigong is relaxing and peace no matter what method you use to practice it. "Relaxing" includes two aspects - physical relaxing and mental relaxing. Physical relaxing is the relaxation from the aspect of joints, muscles and organs, when people are practicing health-care Qigong. And mental relaxing is from physical and spiritual aspect. It is the process from shallow to deep, from internal to external and is the comprehensive relax of body and conscious. "Peace" refers to inside peace without any other distractions so the brain can get enough rest to ensure the effective adjustment of functions of human vegetative nervous system and central nervous system. Thus, human emotion is adjusted. During the practice of health-care Qigong, relaxing and peace supplement each other and they are needed together. (3) the way of imagination. Practice of health-care Qigong is a gradual process, so trainers must deal with the relation of physical and mental health well. Only in that way can the effect of practicing health-care Qigong make use to its greatest extension to improve physical and mental health and adjust human emotion. Impatient practice only gets the opposite effect. Every trainer will get through four stages: laying more emphasis on form than conscious; lay emphasis on both form and conscious; lay more emphasis on conscious than form; no form and conscious. Every stage has its own focus when dealing with the relation of motion and motionlessness and the relation of practicing and keeping. Make sure that every stage are finished well, so the better unity of internal and external cultivation can be achieved and to adjust human emotion is better completed.

\section{The analysis of health-care Qigong in terms of culturology}

There are two points about the analysis of emotional adjustment mechanism of health-care Qigong. (1) the influence of Chinese traditional philosophy to health-care Qigong. Chinese traditional philosophy gives priority to the overall conception of the coordination of human and environment, nature, society and human action and his thought. And Confucianism pay attention to self-improvement - politeness leads heart. Legalism teaches people to be good to suit to the regulation of social development. Taoist thinks that nature is god. All these traditional philosophy made great influence on health-care Qigong. In ancient China, they also focused on cultivation of ethics and the role Qigong played in emotional adjustment. There is a saying in "Shao Lin Qi Gong": "Lao zi keep great gender to rule his men; others practice Qigong to adjust emotion; monks do Qigong to pursue inner peace. That is the way how Yi jin works, all through every corner of your body”. This record is a powerful evidence to prove that practicing health-care Qigong should pay attention to body improvement and mind refinement simultaneously. (2)the influence of traditional Chinese medical (TCM) relation between physical and mental health to health-care Qigong. The major content of seven emotion in Chinese medical, which is the basis of the view that the air is so important to both physics and mentality, is the relation of physics and mentality. Seven emotions in TCM are happiness, anger, sorrow, thinking, sadness, fear and surprise. They are important to the internal organs. If there is something wrong with seven emotions, they may cause the dysfunction or lack of air which may cause the blood problems. Then the whole body will lack of air and blood, so people may feel ill caused by functional disorder and imbalance of yin and yang. TCM thinks the normal way to cure the illness caused by seven emotions is to put right one's behavior, be nice and 
peaceful and not to be interrupted by the outside factors. If the change of seven emotions is not under control of self adjusting system, people may lose their temple and impact their health. Practicing health-care Qigong can cultivate one's taste and teach people to control emotion by sensibility. It can realize the efficient adjustment of human emotion through being peaceful, relaxing and optimistic without distractions.

\section{The analysis of health-care Qigong in terms of psychology}

The below two points are about the analysis of emotional adjustment mechanism of health-care Qigong in terms of psychology. (1) the accumulation of biological energy. The theory about characters, wrote by $\mathrm{W} \cdot$ Leich said that stress is a kind of biological energy which can flow freely through people's body and accumulates increasingly. This kind of energy can lead emotion disorder once the energy accumulated to a certain extent to release or form an obstacle. People in healthy mentality can coordinate the function of releasing or accumulating stress, while people in unhealthy mentality who cannot control the free flow of this kind of biological energy have trouble in releasing the energy. They cannot control their emotion because of the obstacle formed by the excessive energy. Health-care Qigong can help people ease the tension and give optimistic guide to release the accumulated biological energy so as to adjust human emotion. (2) suggestion training. There is certain connection between inner effect and autosuggestion when people are practicing health-care Qigong. The inner effect during the practice of health-care Qigong is objective conscious which make sense in the aspect of conscious and is able to persuade people and give objective suggestions in order to adjust emotion adjustment. But health-care Qigong is different with suggestion training which is to interfere patients with mental disorder or athletes through language, behaviors and setting scenes. Health-care Qigong can only have an effect by adjusting physics and mentality and using conscious to work on both physics and mentality. Only in that way can health-care Qigong reach the effect to influence body to regulate functions and adjust emotions.

\section{Conclusion}

For thousands of years, Chinese Qigong was combined with the essence of Chinese traditional philosophy, literature, the theory of Chinese medicine and religion, etc. during its development and evolution. In recent years, health-care Qigong appears on the basis of Qigong. Health-care Qigong is a kind of national traditional sports which based on the combination of physical activities, inspiration and expiration and psychological adjustment. And health-care Qigong is also called a kind of self -motion. It is initiative exercise with its purpose of fitness. Health-care Qigong can effectively adjust human emotion, improve the function of central nervous system and cultivate morality. Therefore, human emotion can be adjusted effectively.

\section{References}

[1] Zhang Qi. A Brief Introduction to the Influence of Health-care Qigong to Human Health, Journal of Shanxi College of Chinese Traditional Medicine,2009,04:77-78.

[2]Chai Xin. Analysis on Health-care Qigong in View of Philosophy, Journal of Xichang College(Natural Sciences),2010,02:118-119+125

[3]Li Binhong. A Brief Introduction to the Influence of Health-care Qigong to Human Health, Friends of Science,2010,20:105-106.

[4]Ju Xiangyang. A Study on Psychological and Mechanism Effect of Chinese Health-care Qigong, Journal of Hebei Physical Educational Institution,2010,06:87-90.

[5]You Guiying,Wang Chengguo. Analysis of Significance of Health-care Qigong on Building Harmonious Society, Boxing(Martial Science),2012,10:99-101. 
[6]Chen Jiyan. The Influence of Health-care Qigong Courses on Physical and Mental Health Level of University Students, Neijiang Technology,2012,11:62.

[7]Tang Guoliang. Stimulation of Health-care Qigong on Human Spirit,Technology Information, 2012,36:656.

[8]Fanf Tongjie. New Version of Analysis of Effect of Health-care Qigong on Human Body Fitness, New West(Theory Version),2012,10:181+170. 